\title{
VARIATION OF CAPACITIVE REACTANCE OF COUPLED MICROSTRIP LINE STRUCTURE WITH WIDTH OF THE SIMILAR METAL STRIPS
}

\author{
Rajeev Ranjan Kumar and S. P. Singh
}

\begin{abstract}
In the age of digital and analog electronics electrons play a significant role in the growth of various technologies being used in the vast development of nation as well as whole world. Electrons play a dual character of particle as well as waves or radiations. Due to particle characters electrons constitute currents that flow through high quality conductors and superconductor as well. Also these electrons when accelerated to a high energy produce electromagnetic radiations of wide range of frequencies from radio to giga hertz range. With the advent of microwave integrated circuits the large scale components have been reduced to miniaturized size components such as strip line and micro striplines and their variants. Their weight and cost have been largely reduced. Such components are known as planar transmission structures having significant properties such as low attenuation, easily replaceable with increased reliabilities. In the present work we have to study the capacitive reactance of a coupled micro stripline structure which requires the primary study of characteristic impedance and phase velocity. The capacitive reactance is the function of permittivity, height of the dielectric materials, and width of metal strips along with the thickness of metal strip. Present study is focused on the variation of capacitive reactance with the width of similar metal strips.
\end{abstract}

Index Terms Capacitive reactance, Strip width, Permittivity, Phase velocity \& Characteristic impedance.

Reference to this paper should be made as follows:

Rajeev Ranjan Kumar and S. P. Singh, (2020), "VARIATION OF CAPACITIVE REACTANCE OF COUPLED MICROSTRIP LINE STRUCTURE WITH WIDTH OF THE SIMILAR METAL STRIPS" Int. J. of Electronics Engineering and Applications, Vol. 8, No. 2, pp. 22-28, DOI10.30696/IJEEA.VIII.II.2020.22.28

\section{Biological Notes:}

Dr. Rajeev Ranjan Kumar has been working as a Associate Professor in ECE Department at Dr. C $V$ Raman University, Vaishali, Bihar. He obtained B.E. from College of engineering, Osmanabad, Maharastra, India. He has done M. Sc. Engg.. from M.I.T. Muzaffarpur and Ph. D. from M.I.T. Muzaffarpur under BRA Bihar University, Muzaffarpur, Bihar. He has 20+ Years' experience as a Assistant Professor with prestigious organization. He has also been worked as a Joint Co-coordinator of EDUSAT Programme and Assistant Examination Controller. He has published several papers in National and International Journals of repute. He also attended National and international seminars and conferences. He is co-author of (i) Concept of Engineering Physics and (ii) Electromagnetic Field Theory. He is also life member of ISTE and IETE.

Dr. S. P. Singh has been working as Associate professor of Applied Physics at RVSCET, Jamshedpur from 2009. Dr. Singh is currently acting as a Head, Department of Science \& Humanities of the college. He has been awarded Ph.D.from B.R.A.Bihar University, Muzaffarpur in 1999 with the specialization in Radio Physics and Electonics. He has attended more than twenty National and International seminars at Rourkela, Kolkata, Ranchi, Muzaffarpur, Jaunpur etc. He has published several papers in Journals of National and International repute. He is also associated as life member with Purvanchal Academy of Science, Uttar Pradesh, The Indian Physical Society, Kolkata. 


\section{INTRODUCTION}

The electromagnetic energy offers many new and existing possibilities in agriculture, food and seeds preservation and to change vegetable taste by reducing acidity. Further electromagnetic devices include transformers, electric relay, radio/TV, telephones, electric motors, transmission lines, waveguides and lasers. The design of these requires knowledge of the laws and principles of electromagnetic fields. The basic laws of electromagnetic fields are defined by four Maxwell field equations such as

$$
\begin{aligned}
\tilde{\mathbf{N}} . \mathbf{D} & =\rho_{\mathrm{v}} \\
\tilde{\mathbf{N}} . \mathbf{B} & =0 \\
\tilde{\mathbf{N}} \mathbf{X} \mathbf{E} & =-\partial \mathbf{B} / \partial \mathrm{t} \\
\tilde{\mathbf{N}} \mathbf{X} \mathbf{H} & =\mathbf{J}+\partial \mathbf{D} / \partial \mathrm{t}
\end{aligned}
$$

Where, $\square=$ Vector differential operator, $\mathrm{D}=$ Electric flux density, $\mathrm{B}=$ Magnetic flux density, $\mathrm{E}=$ Electric field intensity, $\mathrm{H}=$ Magnetic field intensity, $\rho \mathrm{v}=$ Volume charge density, and $\mathrm{J}=$ Current density.

These field equations deal with vector quantities. These equations are derived from time varying situations of electromagnetic field vectors and these are useful in practical electromagnetic devices. Now the two parallel wire transmission lines are the simplest structures for microwave signals to be transmitted but these are very much lossy in high range of frequency. To minimize the size \& cost new technology known as planar transmission line technology has been developed with the advent of microwave integrated circuits. There are various transmission structures which have been developed \& designed by different pioneers of this field suitable for high range of frequency such as stripline, microstripline, slot line, coplanar strips and coplanar wave guides \& their different variants. The stripline and microstripline are the simplest structure having features: Reduced cost, Miniaturized size, Easily replaceable and Minimized losses compared to other transmission structures.

Several types of microwave striplines have been developed and used as common microwave transmission components. With the advent of microwave integrated circuits, the older transmission structures like two parallel wire lines, waveguide and co-axial line have been revolutionized with the introduction of miniaturized microwave planar transmission structure. This is very attractive and useful for micro wave integrated circuits applications involving a larger number of identical units and requiring a high density of packaging.

In the present work characteristic parameters of coupled micro stripline are to be studied specifically capacitive reactances for even and odd mode wave propagation and their variations with width of similar metal strips. Any transmission structure possesses certain characteristic parameters like characteristic impedance, propagation parameter and phase velocity.

\section{CONFIGURATION OF STRIPLINE AND ITS COUPLED STRUCTURE}

A microstripline consists of a narrow conductor strip on one side of a dielectric substrate and the other side being completely metalized to serve as a ground plane. The microstrip structure may be derived from the stripline configuration by removing the top ground plane and upper laminate with metal strip pasted on substrate. 
When two similar striplines are placed with sides parallel to each other, coupled structure is formed. This is most useful and widely applied in the field of microstripline structure in the design of stripline coupler and directional coupler. The structure and field configurations is as shown in fig- 1 and its field configurations for even and odd mode are shown in fig-2 and fig-3.

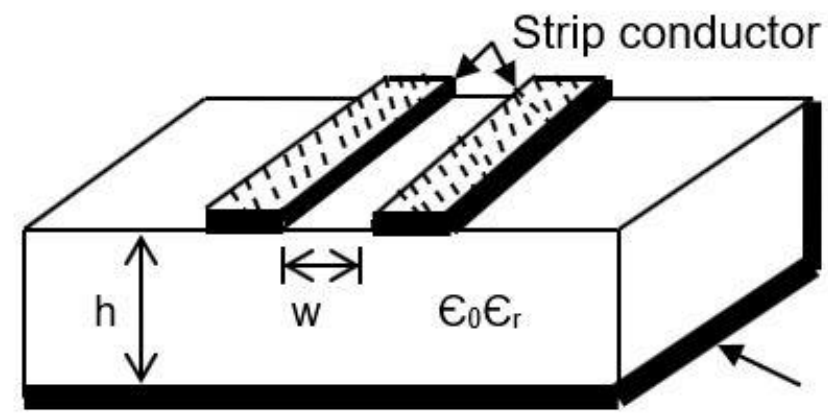

Ground plane

Fig. 1. Couple stripline structure

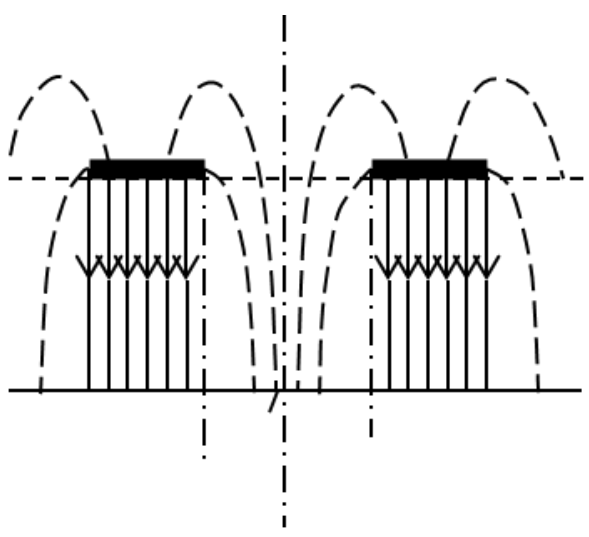

Fig. 2. The Even-mode

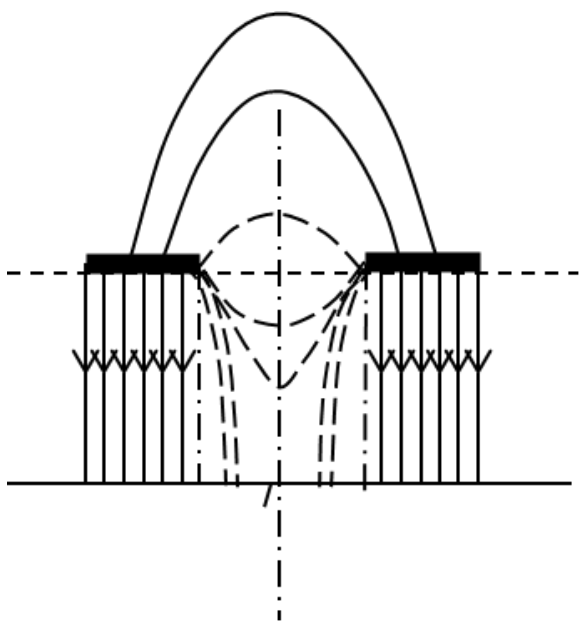

Fig. 3. The Odd Mode

When energy is fed to the coupled structures wave propagates in both striplines. There is interaction between the fields produced by the waves. When wave propagates in the same direction, forward 
coupling occurs and reverse coupling occurs when they propagates in opposite direction. Even and odd mode wave propagation is considered.

\section{FORMULATION OF CHARACTERISTICS IMPEDANCE FOR COUPLED STRUCTURES}

The characteristic impedance can be calculated with the help of elementary transmission line equation expressed as

$\mathrm{Z}_{\mathrm{o}} \quad=\quad 1 / \mathrm{V}_{\mathrm{P}} \mathrm{C}_{\mathrm{P}}$

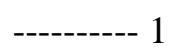

Where, $\mathrm{V}_{\mathrm{P}} \quad=$ phase velocity of the wave traveling along the microstrip line.

$$
=\mathrm{c} / \ddot{\mathrm{O}} \hat{\mathrm{I}}_{\mathrm{reff}}
$$

$\mathrm{C}_{\mathrm{P}} \quad=$ capacitance per unit length of the line, which depends on geometry and dielectric medium of the structure and expressed as

$$
\mathrm{C}_{\mathrm{p}}=\left(\hat{\mathrm{I}}_{\mathrm{reff}} / \mathrm{c} . \eta\right)(\mathrm{w} / \mathrm{h})+(2 / 3)\left(\hat{\mathrm{I}}_{\mathrm{reff}} / \mathrm{c} . \eta\right)(\mathrm{w} / \mathrm{h})\left(\hat{\mathrm{I}}_{\text {reff }} / \mathrm{c} . \eta\right) \cdot(2.7 / \log 4 \mathrm{~h} / \mathrm{t})
$$

Here, $c=$ velocity of electromagnetic wave in vacuum,

$\eta=377 \Omega=$ Intrinsic impedance,

$\mathrm{h}=$ height of the substrate \&

$\mathrm{w}=$ width of the metal stip.

$\hat{\mathrm{I}}_{\text {reff }}=$ Effective dielectric constant, Using VP and CP in equation 1, Z0 is expressed as

$\mathrm{Zo}=\left(\mathrm{h} / \mathrm{Ö}_{\mathrm{reff}}\right) \cdot[1 /[(\mathrm{w} / \mathrm{h})+(2 \mathrm{w} / 3 \mathrm{~h})+(2.7 / \log 4 \mathrm{~h} / \mathrm{t})]$

Due to inhomogeneity the phase velocity $\left(\mathrm{V}_{\mathrm{P}}\right)$ for the isolated case is replaced by Vpe for the evenmode and $\mathrm{V}_{\mathrm{po}}$ for the odd- mode when coupled structure is considered. Further in place of $\hat{\mathrm{I}}_{\text {reff }}$ the effective dielectric constants $\left(\hat{\mathrm{I}}_{\text {reff }}\right)_{\mathrm{e}}$ and $\left(\hat{\mathrm{I}}_{\text {reff }}\right)_{\mathrm{o}}$ are to be used for even- and odd- modes respectively in coupled structure.

Similarly, $Z_{\mathrm{oe}}$ and $\mathrm{Z}_{\mathrm{oo}}$ represent the characteristic impedances for even-and odd- modes respectively. Where

Zoe $=\left(\eta / \sqrt{ } \epsilon_{\text {reff }}\right) \cdot\left[1 /\left\{(w / h)\left[1+\left(1 / 3 / \sqrt{ } \epsilon_{\text {reff }}\right)\right]+\left(1 / 3 \sqrt{ } \epsilon_{\text {reff }}\right)(1 /(w / s)+1)\right\}\right.$ 5

And

Zoo $=\left(\eta / \sqrt{ } \epsilon_{\text {reff }}\right) .\left[1 /\left\{(w / h)\left[1+\left(1 / 3 \sqrt{ } \epsilon_{\text {reff }}\right)+\left(4 / 3 \sqrt{ } \epsilon_{\text {reff }}\right)(1 /(\mathrm{s} / \mathrm{w})+1)\right\}\right]\right.$

Here, s denotes the spacing between two metal strips.

\section{FORMULATION OF PHASE VELOCITY FOR COUPLED STRUCTURE}

As there are two modes of wave propagation such as even and odd through coupled structure, so the velocity of propagation of wave through structure is also of two types. These are even-mode velocity and odd-mode velocity denoted by (Vp)e and (Vp)e respectively. These are expressed as 
For even mode

$(\mathrm{Vp}) \mathrm{e}=\mathrm{c} /(\sqrt{ }$ Creff)e

and for odd mode

$(\mathrm{Vp}) \mathrm{o}=\mathrm{c} /(\sqrt{ }$ Greff $) \mathrm{o}$

\section{FORMULATION OF CAPACITIVE REACTANCE OF COUPLED STRUCTURE}

As in the conformal transformation microstrip geometry is converted into parallel plate geometry, the microstrip structure is equivalent to a parallel plate capacitor filled with dielectric substrate. The capacitance between two plates is the ratio of total electric flux to the potential difference between the plates. This capacitance is the function of geometry of the plates, spacing between two plates and dielectric medium. The capacitance per unit length of microstrip structure is related with the characteristic impedance $\&$ phase velocity of the wave propagating through the microstrip structure and is given as

$$
\mathrm{C}_{\mathrm{P}}=\frac{1}{\mathrm{~V}_{\mathrm{P}} \cdot \mathrm{Z}_{\mathrm{O}}}
$$

Where,

$$
\begin{array}{ll}
\mathrm{V}_{\mathrm{P}} & =\text { phase velocity of the wave traveling along the microstrip line. } \\
& =\mathrm{c} / \sqrt{ } \epsilon_{\text {reff }} \\
\mathrm{c} & =\text { Velocity of electromagnetic wave in free space. } \\
\mathrm{G}_{\text {reff }} & =\text { Effective dielectric constant } \\
& \approx(\mathrm{Cr}+1) / 2 \\
\mathrm{CP} & =\text { capacitance per unit length of the line. } \\
\mathrm{Zo} & =\text { Characteristic impedance }
\end{array}
$$

But in case of coupled microstripline structure, there exists two capacitors having same separation between them. The electric field configurations between these capacitors get modified. This further modifies the capacitance of the structure. Also power flowing in one line gets coupled to the other line \& either in same direction or in opposite direction constituting two modes of wave propagation through the coupled structure namely: (i) even-mode of propagation and (ii) Odd-mode of propagation.

Hence in case of coupled microstripline structures there are two types of capacitances

(i) $\mathrm{Cpe}=$ Capacitance of coupled structure for even-mode of propagation

$$
\mathrm{C}_{\mathrm{Pe}}=\frac{1}{\mathrm{~V}_{\mathrm{Pe}} \cdot \mathrm{Z}_{\mathrm{oe}}}
$$

Where,

$$
\begin{aligned}
& \mathrm{Z}_{\mathrm{oe}}=\text { Even-mode characteristic impedance } \\
& \mathrm{V}_{\mathrm{pe}}=\text { Even-mode phase velocity }
\end{aligned}
$$

Even mode capacitive reactance $\left(\mathrm{X}_{\mathrm{CE}}\right)=1 / \omega \mathrm{C}_{\mathrm{Pe}}$

$$
\begin{gathered}
=1 / \text { angular frequency } \times \text { Even mode Capacitance } \\
=Z_{0 \mathrm{e}} V_{\mathrm{Pe}} / 2 \pi \mathrm{f} .
\end{gathered}
$$


(ii) $\mathrm{C}_{\mathrm{po}}=$ Capacitance of coupled structure for odd-mode of propagation

$$
\mathrm{C}_{\mathrm{Po}}=\frac{1}{\mathrm{~V}_{\mathrm{Po}} \cdot \mathrm{Z}_{\mathrm{oo}}}
$$

Where,

Zoo = Even-mode characteristic impedance

$\mathrm{Vpo}=$ Even-mode phase velocity

Odd mode capacitive reactance $(\mathrm{XCO})=1 / \omega \mathrm{C}_{\mathrm{Po}}$

$$
\begin{aligned}
& =1 / \text { angular frequency x Odd mode Capacitance } \\
& =\mathrm{Z}_{0 \mathrm{o}} \mathrm{V}_{\mathrm{Po}} / 2 \pi \mathrm{f} .
\end{aligned}
$$

Obviously, these reactances depend on strip geometry, spacing between two strips \& effective relative permittivity of the medium which is Alumina $\left(\epsilon_{r}=9.6\right)$.

\section{STUDY OF VARIATION OF EVEN \& ODD-MODES CAPACITIVE REACTANCE OF COUPLED STRUCTURE WITH METAL STRIP WIDTH}

The capacitive reactances for even and odd modes are important parameters of the coupled structure for storing the energy. They can also give out the energy to other circuits during discharging. The study of variation of these capacitive reactances with stripwidth has been carried out manually. By giving different values of stripwidth different values of even and odd-modes capacitive reactances have been calculated. Keeping stripwidth along $\mathrm{x}$-axis and capacitive reactances along $\mathrm{y}$-axis graphs have been plotted as shown in graph -1. From these graphs it is evident that with increase of stripwidth both even and odd mode capacitive reactances decrease. The odd-mode capacitive reactance is smaller than that of even-mode capacitive reactance. It is also evident from the result that even mode capacitive reactance has faster decrease rate than the decrease rate of odd mode capacitive reactance.

Graph - 1: Study of Variation of even \& odd-modes Capacitances of

coupled microstripline with stripwidth

$$
\begin{aligned}
& \mathrm{h}=100 \mathrm{mils}, \mathrm{t}=0.05 \mathrm{mils}, \mathrm{f}=2 \mathrm{GHz}, \mathrm{s}=20 \mathrm{mils} \\
& \epsilon_{\mathrm{r}}=9.6,1 \mathrm{mils}=2.4 \times 10-3 \mathrm{~cm}
\end{aligned}
$$

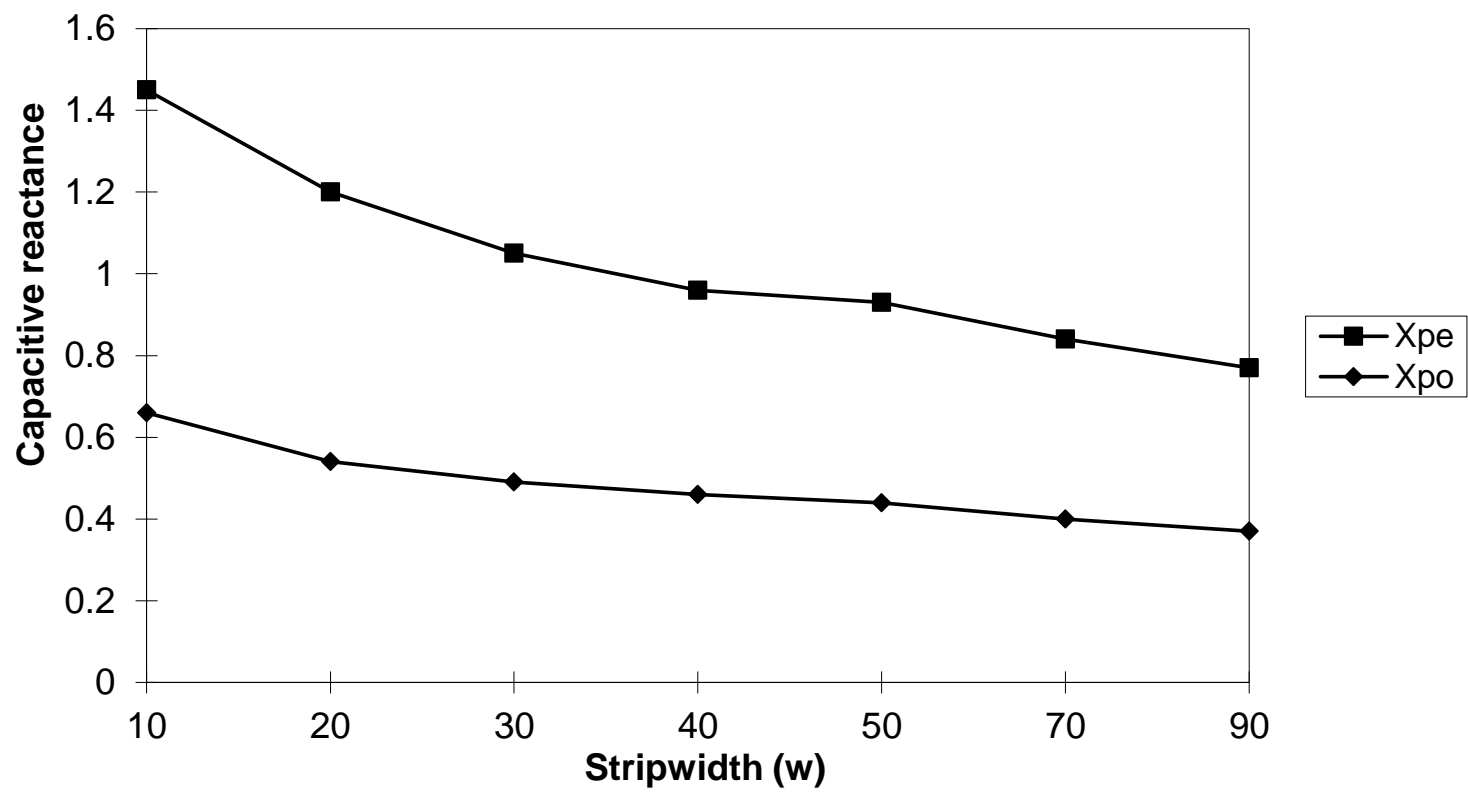




\section{CONCLUSIONS}

In coupled structure the equivalent capacitive reactance under study is due to capacitances existing between two strip lines as well as between top $\&$ bottom of the substrates. So this capacitive reactance solely \& wholly depends on geometry of the coupled structure as well as dielectric medium used. With the increase of width of strip the energy and electric flux storing capacity increases. This property of the coupled structure can be utilized in designing microstripline filter, frequency generator and directional coupler. It may carry out future study.

\section{REFERENCES}

[1] H. Howe, "Stripline Design", Artech House, Dedham mass USA, 1974,p. 111.

[2] H. Howe, "Stripline circuit design of coupled Parallel lines", Artech House, Dedham mass USA, 1974, pp. 112-137.

[3] T. C. Edwards, "Foundation for Microstrip Circuit Design", John Wiley and Sons, 1976, p. 134.

[4] B. Bhat and S. K. Koul, "Stripline Like Transmission Line for MIC's", Wiley Eastern Limited, India, 1990.

[5] H.A. Wheeler, "Transmission Line Properties of Parallel Wide Strip by Conformal Mapping Approximation", IEEE Transactions on MTT, vol.12, 1964.

[6] H.A. Wheeler, "Transmission Line Properties of Parallel Strip Separated by Dielectric Sheet”, IEEE Transactions on MTT, vol. 13, 1965.

[7] K. C. Gupta, R. Garg and S. J. Bahl, "Microstrip and Slot Lines", Artech House, Inc. [8] K. C. Gupta and A. Singh, "Microwave Integrated Circuits", Wiley Eastern Pvt Limited.

[9] B. Easter and K. C. Gupta, "More Accurate Model of the Coupled Microstriplines Sections", IEE Journal on Microwave, vol. 3, no. 3, 1973, p. 79.

[10] T. G. Bryat and J. A. Weiss, "Parameters of Microstriplines and of Coupled Pairs of Microstrip Line”, IEEE Transactions on MTT, vol. 16, No. 12, 1968.

[11] A. Schwarzmann, "Approximate Solutions of Coupled Pair of Microstrip Line in MICs Microwave", Journal, vol. 12, no. 5, 1969, p. 79.

[12] M.K.Krage and G.I.Haddad, "Characteristic of coupled microstrip transmission line- 1", Coupled mode formulation”, IEEE Trans. MTT, vol. 4, no.18, 1969.

[13] M.K.Krage and G.I.Haddad, "Characteristic of coupled microstrip transmission line- 2, Evolution of coupled line parameter", IEEE Trans. MTT, vol. 15, April 70.

[14] Fooks E. H., "Microwave engineering using microstrip circuits", Prentice Hall, New York, 1990.

[15] Gardiol Fred E., "Microstrip circuits", Wiley, New York, 1994.

[16] Krowne, C. M., "Dyadic Green's function modifications for obtaining attenuation in microstrip transmission layered structure with complex media", IEEE Trans. MTT, Vol. 50, Jan 2002

[17] Pozar, David M. Microwave Engineering Addison-Wesley Publishing Company. ISBN 978-81 265-4190-4, 2017.

[18] Lee, T. H., "Planar Microwave Engineering”, Cambridge University Press, 2004. 